Nonlinear Processes in Geophysics (2005) 12: 101-115

SRef-ID: $1607-7946 / \mathrm{npg} / 2005-12-101$

European Geosciences Union

(C) 2005 Author(s). This work is licensed

under a Creative Commons License.

\title{
Quasiadiabatic description of nonlinear particle dynamics in typical magnetotail configurations
}

\author{
D. L. Vainchtein ${ }^{1,2}$, J. Büchner ${ }^{3}$, A. I. Neishtadt ${ }^{1}$, and L. M. Zelenyi ${ }^{1}$ \\ ${ }^{1}$ Space Research Institute, ul.Profsouznaya, 84/32, Moscow, Russia, GSP-7, 117997 \\ ${ }^{2}$ Department of Mechanical \& Environmental Engineering, University of California, Santa Barbara, CA 93106, USA \\ ${ }^{3}$ Max Planck Institut für Aeronomie, Max-Planck-Str. 2, D-37191 Katlenburg-Lindau, Germany \\ Received: 2 March 2004 - Revised: 22 November 2004 - Accepted: 7 January 2005 - Published: 27 January 2005 \\ Part of Special Issue "Advances in space environment turbulence"
}

\begin{abstract}
In the present paper we discuss the motion of charged particles in three different regions of the Earth magnetotail: in the region with magnetic field reversal and in the vicinities of neutral line of $X$ - and $O$-types. The presence of small parameters (ratio of characteristic length scales in and perpendicular to the equatorial plane and the smallness of the electric field) allows us to introduce a hierarchy of motions and use methods of perturbation theory. We propose a parameter that plays the role of a measure of mixing in the system.
\end{abstract}

\section{Introduction}

In recent years there has been much interest in the nonlinear dynamics of charged particles in various regions of the earth magnetotail. This research started with the papers (Northrop, 1963; Speiser, 1965, 1967; Sonnerup, 1971; Lyons and Speiser, 1982). Since then, the motion of particles in the magnetic field reversal configurations was studied by different authors, both numerically and analytically for quite different approximations of the magnetic field geometry. The most basic, one-dimensional, approximation was discussed in many publications (see Chen (1992) and references therein). Longitudinal variations of the magnetic field were first taken into consideration analytically in Zelenyi et al. (1990) and numerically in Karimabadi et al. (1990), where the problem was considered for a variety of configurations of magnetic field.

The motion of particles in the vicinity of an $X$-type neutral line was studied theoretically and numerically (Martin, 1986; Burkhart et al., 1991; Moses et al., 1993), numerically by (Bruhwiler and Zweibel, 1992; Smets et al., 1993; Nocera et al., 1996). The impact of the electric field was studied analytically and numerically in Deeg et al. (1991) and numerically in Petkaki and Mackinnon (1994). The dynamics

Correspondence to: D. L. Vainchtein

(dmitri@engineering.ucsb.edu) in the vicinity of an $O$-type neutral line was studied numerically in Martin et al. (1991) and analytically in Larson and Tracy (1993).

It is known that for a wide range of parameters the motion of charged particles in the magnetotail is chaotic (Chen and Palmadesso, 1986; Büchner and Zelenyi, 1989), see also Chen (1992) for a review. It was shown by Büchner and Zelenyi (1989), that in the domain where the Larmor radius of charged particles is much larger than the smallest radius of curvature of magnetic field lines the jumps of an adiabatic invariant lead to chaotic behaviour. In closed systems chaotic behaviour necessarily leads to complete mixing in the accessible part of the phase space. However, in the real magnetotail the electric field accelerates particles, thus limiting the time they spend in the magnetotail, which, in turn, defines how much mixing can happen before the particles leave the system.

One of the standard methods of studying the chaotization and mixing (in both analytical and numerical approaches) is to look at the behaviour of approximate invariants of a system. The action variable $I_{z}$ (the action variable of the motion perpendicular to the equatorial plane), that is a standard action variable of the Hamiltonian dynamics and analogous to the the magnetic moment $\mu$ in the guiding center theory, was introduced by Speiser (1970). The important role played by $I_{z}$ was first recognized by Sonnerup (1971). That single action variable is, naturally, not sufficient for a complete description of more realistic models, which include both variations of parameters of the magnetic field along the equatorial plane and the influence of the electric field. In Zelenyi et al. (1990) new results were obtained by introducing an additional, longitudinal, adiabatic invariant, that is analogous to $J_{\|}$ (the action associated with the motion along a magnetic field line) in the guiding center theory. This approach proved to be quite useful and allowed the authors to obtain important estimates of the particles' acceleration. The method was based on two consecutive averagings of the equations of motion, and the validity of the approach, that may be non-trivial (because of separatrix crossings, even the adiabatic invariant of 
the fastest motion, $I_{z}$, is not well-conserved) were not discussed. The situation here is quite different from the case when the guiding center theory works. In the latter case, two consecutive averagings of the equations of motions are clearly valid and the adiabatic invariants are preserved for timescales growing exponentially with the magnetic field intensity (see Benettin and Sempio, 1994).

In the present paper we consider the motion of charged particles in three distinct regions of the Earth magnetotail: in the region with magnetic field reversal (MFR), taking into consideration the longitudinal variations of the magnetic field, and in the vicinities of neutral lines of $X$ - and $O$-type (XO). In all three regions the magnetic field was considered two-dimensional (no cross-tail component) and the presence of the constant electric field was taken into account. The are several objectives of the present publication. First, our goal is to define what results obtained for uniform normal component of the magnetic field, $B_{n}$, are applicable for $x$-dependent $B_{n}$ and what phenomena are qualitatively different. Second, we would like to obtain the conditions under which the mixing in the phase space is not too strong. We propose a new parameter that describes the extent of mixing in the system (see Sect. 5). This parameter depends on the magnitude of the electric field and the smoothness and the curvature of the magnetic field. Depending on the value of this parameter, the jumps of the adiabatic invariants destroy original structures in the distribution function or particles accelerate and leave the tail quickly enough to keep the distribution function relatively intact. And finally, we use the technique developed in Büchner and Zelenyi (1986, 1989); Zelenyi et al. (1990) to study the motion of charged particles near neutral lines of $X$ - and $O$-types in order to understand the impact of processes that occur in the vicinities of neutral lines on the overall picture of particles acceleration.

There are two basic phenomena that may affect the picture constructed in the present paper: the time-dependent perturbations (like electromagnetic waves) and the presence of the additional cross-tail component of the magnetic field. The impact of the electromagnetic waves on the dynamics of particles in the magnetotail was studied started in Cattell et al. (1995); Ma and Summers (1998). A range of parameters for which the structure obtained for stationary field configurations survive in the presence of the electromagnetic waves was obtained analytically in Vainchtein et al. (2004). The role of the cross-tail component of the magnetic field in the MFR region was first discussed in Karimabadi et al. (1990) and later studied, both analytically and numerically, in many papers, including Büchner and Zelenyi (1991); Zhu and Parks (1993); Chapman and Rowlands (1998); Ynnerman et al. (2000); Tsalas et al. (2001). It was shown that for a certain range of the ratios of the typical values of the components of the magnetic field the structures obtained for two-dimensional model stay qualitatively the same even in the presence of the third component.

The structure of the paper is as follows. In Sect. 2 we describe the basic models for all the configurations of electromagnetic fields that we use the rest of the paper. In Sect. 3 we discuss the properties of the fastest (perpendicular to the equatorial plane) motion. We show that dynamics is exactly the same for MFR and XO problems. In Sect. 4 we illustrate the averaging method and provide the description of the phase portraits of the averaged system. In Sect. 5 we describe the long-time properties of the particles' dynamics (on times of order of the lifetime of particles in the magnetotail) and introduce a parameter that can be used as a measure of mixing. In Sects. 6 and 7 we derive the acceleration rates in the adiabatic case in the MFR and $X O$ problems. Section 8 contains conclusions.

\section{Main equations}

We start with a derivation of the Hamiltonian of a charged particle in different regions of the Earth magnetotail. We separate three distinct regions: the region with magnetic field reversal (referred as MFR below), and the vicinities of neutral lines of $X$ - and $O$-types. The descriptions of the motion of charged particles near the neutral lines of two types are mathematically very similar. We denote by XO the equations applicable to the dynamics near either neutral line and provide the separate descriptions when necessary.

We use the following models of the magnetic field:

$\mathbf{B}=-B \frac{z}{L_{z}} \mathbf{e}_{x}+B_{n}(x) \mathbf{e}_{z} \quad(M F R)$,

$\mathbf{B}=s B \frac{z}{L_{z}} \mathbf{e}_{x}+B \frac{x}{L_{x}} \mathbf{e}_{z} \quad(X O)$.

In Eq. (1), $\mathbf{e}_{i}$ are the unit vectors of a Cartesian coordinate system in which the $\mathrm{x}$-axis is directed towards the earth, the $y$-axis is in the equatorial plane and is directed from dusk to dawn and the z-axis is perpendicular to the equatorial plane and is directed from south to north; $L_{x}$ and $L_{z}$ are the characteristic length scales in $x$ - and $z$-directions, respectively; $s$ is a parameter that is equal to +1 for the problem for an $X$-line and is equal to -1 for the problem for an $O$-line.

The electric field is induced by the solar wind and has the form

$$
\mathbf{E}=E \mathbf{e}_{y} .
$$

In the magnetotail $E$ can reach the values of order of $0.1 \mathrm{mV} / \mathrm{m}$. These values of $E$ correspond to a total potential drop of order of $25 \mathrm{kV}$. The latter quantity gives the upper boundary for the energy that a particle can gain in the magnetosphere due to the acceleration in a dawn-dusk electric field.

For the MFR problem the specified configuration of the electromagnetic field is schematically shown in Fig. 1a and can be described by a vector potential

$$
\mathbf{A}=\left(0 ; \int_{x_{0}}^{x} B_{n}(\zeta) d \zeta-B \frac{z^{2}}{2 L_{z}}-c E t ; 0\right)
$$

where $x_{0}$ is an arbitrary constant (it corresponds to an ambiguity in choosing a vector potential), $t$ is the time and $c$ is the 

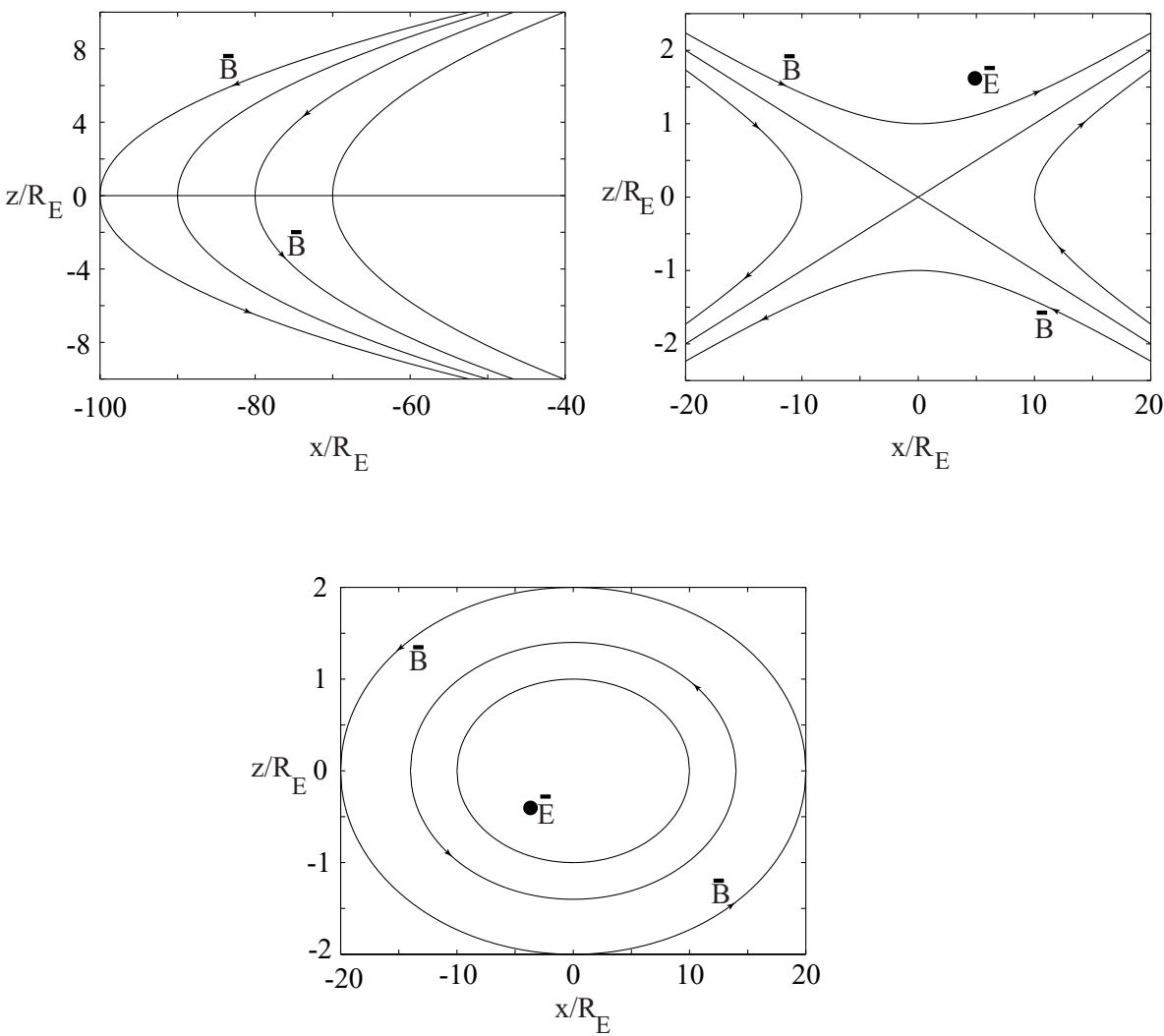

Fig. 1. Characteristic configuration of electromagnetic field: (a) In the MFR region, (b) in the vicinity of a neutral line of $X$ type, and (c) in the vicinity of a neutral line of $O$ type

speed of light. In the specified gauge, the Hamiltonian of a charged particle is

$H=\frac{1}{2 m}\left[P_{x}^{2}+\left(P_{y}-\frac{e}{c}\left(\bar{B}_{n} \int_{x_{0}}^{x} \tilde{\beta}(\zeta) d \zeta-B \frac{z^{2}}{2 L_{z}}-c E t\right)\right)^{2}+P_{z}^{2}\right]$,

where $\bar{B}_{n}$ is some typical value of $B_{n}(x)$ and $\tilde{\beta}(x)=B_{n}(x) / \bar{B}_{n}$.

Note, that the time dependence in (2) is just a standard pay off for using a vector potential and the Hamiltonian form of equations of motion (see e.g. Landau and Lifshitz, 1959) and is not related to time-dependent field reversal configurations similar to those considered in Chapman (1994); Chapman and Watkins (1996).

The configurations of electromagnetic fields in the vicinity of $X$ - and $O$-lines are shown in Figs. $1 \mathrm{~b}$ and c, respectively. The vector potential can be written as

$$
\mathbf{A}=\left(0 ; s B \frac{x^{2}}{2 L_{x}}-B \frac{z^{2}}{2 L_{z}}-c E t ; 0\right)
$$

and the Hamiltonian of a charged particle is

$$
H=\frac{1}{2 m}\left[P_{x}^{2}+\left(P_{y}-\frac{e}{c}\left(s B \frac{x^{2}}{2 L_{x}}-B \frac{z^{2}}{2 L_{z}}-c E t\right)\right)^{2}+P_{z}^{2}\right]
$$

In Eqs. (2) and (3), $\mathbf{P}=\left(P_{x}, P_{y}, P_{z}\right), m$ and $e$ are the generalized momentum, the mass and the charge of a particle respectively. As $H$ does not depend on $y$ explicitly, $P_{y}$ is an integral of motion: $P_{y}=P_{y, 0}=$ const.

Introduce dimensionless variables

$\mathbf{x}_{\mathbf{1}}=\mathbf{x} / \sqrt{\rho_{L} L_{z}}, \mathbf{P}_{\mathbf{1}}=\mathbf{P} / m v, t_{1}=t v / \sqrt{\rho_{L} L_{z}}, h=H / m v^{2}$,

where $v$ is a typical velocity of a particle and $\rho_{L}=\mathrm{cmv} / \mathrm{eB}$ is the Larmor radius. Introduce a scaled time:

$$
\tau=\varepsilon t_{1}+P_{y, 0},
$$

where $\varepsilon$ is the normalized electric field:

$$
\varepsilon=\frac{c E}{v \bar{B}_{n}} \quad(M F R), \quad \varepsilon=\frac{c E}{v B} \frac{L_{z}}{\sqrt{L_{z} \rho_{L}}} \quad(X O) .
$$

Estimates of the magnitude of $\varepsilon$ (based on representative values of $E=0.1 \mathrm{mV} / \mathrm{m}, B_{n}=1 \mathrm{nT}$, and $v=700 \mathrm{~km} / \mathrm{sec}$, see e.g. (Kivelson and Russell, 1995)) yield $1 / 10<\varepsilon<1 / 3$. Note, that although the values of $\varepsilon$ are not too small, it is the product of the normalized magnitude of the electric field, $\varepsilon$, and the parameter of the nonuniformity of the $x$ - component of the 
magnetic field ( $v$ for MFR problem, $\lambda$ for the XO problem, see below) that defines the ratio of the characteristic time scales. And those products are sufficiently small. Therefore, the new time $\tau$ can be considered to be slow. In the new variables Hamiltonians (2) and (3) have the form

$h=\frac{1}{2}\left[P_{x_{1}}^{2}+\left(\kappa \int_{x_{1,0}}^{x_{1}} \tilde{\beta}(\zeta) d \zeta-\frac{1}{2} z_{1}^{2}-\kappa \tau\right)^{2}+P_{z_{1}}^{2}\right](M F R)$,

$h=\frac{1}{2}\left[P_{x_{1}}^{2}+\left(s \frac{1}{2} \lambda^{2} x_{1}^{2}-\frac{1}{2} z_{1}^{2}-\tau\right)^{2}+P_{z_{1}}^{2}\right] \quad(X O)$.

In (5) and (6),

$$
\kappa=\frac{\bar{B}_{n}}{B} \sqrt{L_{z} / \rho_{L}} \quad(M F R),
$$

and

$$
\lambda^{2}=\frac{L_{z}}{L_{x}} \quad(X O) .
$$

Parameters $\kappa$ and $\lambda$, that is equivalent to $b_{n}$ from Burkhart et al. (1991), play a crucial role in the description of the dynamics of charged particles.

Now let's consider two problems separately.

\subsection{The Hamiltonian in MFR}

Let us start with the MFR problem. For constant $B_{n}$, the Hamiltonian is time-independent in the moving coordinate frame $\widehat{x}=x-t c E / B_{n}$ (de Hoffman - Teller substitution, de Hoffman and Teller, 1950). One of the main objectives of the present paper is to define what results obtained for uniform $B_{n}$ (see Büchner and Zelenyi, 1989; Zelenyi et al., 1990) are applicable for $x$-dependent $B_{n}$ and what phenomena are qualitatively different.

If $B_{n}$ depends on $x$ smoothly then the Hamiltonian depends smoothly on time. If $B_{n}$ does not change the sign, we can introduce

$$
x_{2}=\int_{x_{1,0}}^{x_{1}} \tilde{\beta}(\zeta) d \zeta
$$

as a new variable. Define a dimensionless parameter $v$ which characterizes the smoothness of $B_{n}(x)$ such that $\beta\left(v x_{2}\right)=\tilde{\beta}(x)$ and $(1 / \beta)\left(\partial \beta\left(v x_{2}\right) / \partial\left(v x_{2}\right)\right) \sim 1$. Hamiltonian (5) gets the form

$$
h=\frac{1}{2}\left[P_{x}^{2} \beta^{2}(v x)+P_{z}^{2}+\left(x^{\prime}-\frac{z^{2}}{2}\right)^{2}\right],
$$

where

$$
x^{\prime}=\kappa(x-\tau) .
$$

In Eq. (8) and below, we do not write subscripts of $x, P_{x}$ and $z, P_{z}$.
For the parameter $\kappa$ introduced in (7) we have $\kappa^{2} \equiv \rho_{c} / \rho_{L}$, where is $\rho_{c}$ is a characteristic minimal curvature radius of a magnetic field line. Recall, that to define $\kappa$ we used some the quantity $\bar{B}_{n}$, which is typical value of the $B_{n}(x)$ for a region where the motion is localized. Therefore, $\kappa$ is constant. It was shown by Wagner et al. (1979), that if $\kappa \gg 1$, the particles are magnetized and their motion can be described by the guiding center theory (GCT). In the magnetotail, electrons are always magnetized, except for the very close vicinity of the $X$-line. The value of $\kappa$ for ions strongly depends on what part of the tail we study and can be anywhere from $\kappa \ll 1$ in the distant tail to $\kappa \gg 1$ near the earth with all the stops in between. Like electrons, ions are magnetized if $\kappa \gg 1$. Up to the best of our knowledge, there is no complete analytical picture of the dynamics if $\kappa \sim 1$ (see e.g. (Delcourt and Martin, 1999) and references therein for progress in research in that region). We are interested in the case $\kappa \ll 1$ with other parameters satisfying the following conditions:

$$
v \leq \kappa \ll 1, \quad \varepsilon \ll 1 .
$$

The physical meaning of $\nu \leq \kappa$ is that $B_{n}(x)$ does not change too much over the amplitude of the $x$-motion. In particular, it excludes from the consideration all the trajectories that come too close to the earth. These inequalities are valid approximately further then $10 R_{E}$ from the earth, where $R_{E}$ is the radius of the earth. In this approximation there is a hierarchy of motions. The fastest motion is on the $\left(z, P_{z}\right)$ plane (i.e. the motion perpendicular to the equatorial plane), the motion on the $\left(x, P_{x}\right)$ plane (the motion towards - away from the earth) is slower and on the $(t, h)$ (acceleration) is the slowest.

Let us now comment on the approximations used in the present paper. First, we neglected the cross-tail component of the magnetic field, $B_{y}$, which was first discussed in Karimabadi et al. (1990) and later studied, both analytically and numerically, in many papers, including Büchner and Zelenyi (1991); Zhu and Parks (1993); Chapman and Rowlands (1998); Ynnerman et al. (2000); Tsalas et al. (2001). It was shown that for a certain range of the ratios of the typical values of the components of the magnetic field the structures obtained for two-dimensional model stay qualitatively the same even in the presence of the third component. One way to take $B_{y}$ into account is to modify the definition of $\kappa$ to

$$
\kappa=\frac{\bar{B}_{n}}{B} \sqrt{L_{z} / \rho_{L}}\left(1+\left(\frac{B_{y}}{\bar{B}_{n}}\right)^{2}\right)^{3 / 4} .
$$

For realistic values of $B_{y}$ (of order of $B_{n}$ ) the value of $\kappa$ would increase approximately twice, remaining smaller than unity. However, further increase of $B_{y}$ does result in chaotization of ion dynamics - the way it should be for $\kappa$ of order 1 . For even larger values of $B_{y}$, the ions are magnetized and we could use guiding center theory instead of the quasiadiabatic theory.

Another nuance is that definition (7), derived in Büchner and Zelenyi (1989) for thick sheets $\left(L_{z}>\rho_{L}\right)$ should be used 

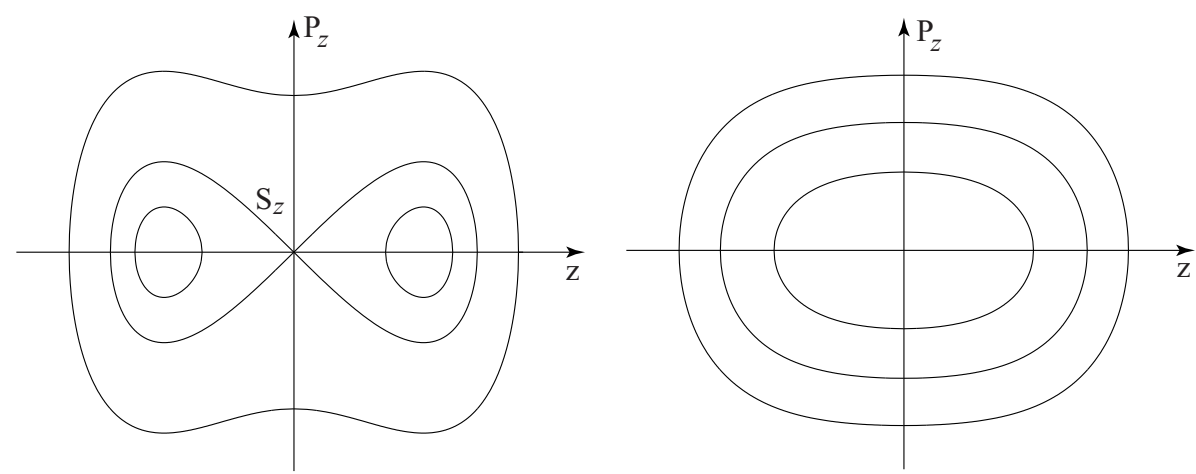

Fig. 2. Characteristic phase portrait of the $z$-motion for (a) $x^{\prime}>0$ and (b) $x^{\prime}<0$.

with care for thin sheets $\left(L_{z}>\rho_{L}\right)$. As was shown in later papers (Savenkov et al., 1991, 1997) for thin sheets the definition of $\kappa$ becomes $\kappa=\bar{B}_{n} / B$. Hence, as it was pointed by Chen (1992) the dynamics of ions depends on two parameters $\bar{B}_{n} / B$ and $L_{z} / \rho_{L}$. The latter parameter contains the energy of particles, $\mathrm{H}$, and when $H \rightarrow \infty, L_{z} / \rho_{L} \rightarrow 0$, but the value of $\kappa$ stays at a fixed value $\kappa=\bar{B}_{n} / B$. In this case one could use a bilinear model of tail field, where particle motion is easily integrable. However, the methods used in the present study are applicable for either definition of $\kappa$, in other words regardless of the relation between $L_{z}$ and $\rho_{L}$.

\subsection{The XO Hamiltonian}

The motion of charged particles in the vicinity of the neutral lines of $X$ - and $O$-types is described by Hamiltonian (6). The typical values of $x$ are of order $\lambda^{-1}$ and the typical values of $z$ are of order 1. Following Martin (1986), we assume $\lambda$ to be of order of $0.1-0.2$, which does not contradict to available experimental data. Therefore, we have the same hierarchy of motions as in the MFR case. For XO we denote

$$
x^{\prime}=s \frac{1}{2} \lambda^{2} x^{2}-\tau,
$$

and Hamiltonian (6) takes the form

$$
h=\frac{1}{2}\left[P_{x}^{2}+P_{z}^{2}+\left(x^{\prime}-\frac{z^{2}}{2}\right)^{2}\right] .
$$

For particles residing near $X$-line, a similar Hamiltonian was considered in Martin (1986); Moses et al. (1993) and (without the electric field) in Burkhart et al. (1991).

\section{The motion on the $\left(z, P_{z}\right)$ plane}

The ratio of typical frequencies for $z$ - and $x$-motion is of order $1 / \kappa \gg 1$ (MFR) (in the part of the tail we are interested in) or of order $1 / \lambda \gg 1(\mathrm{XO})$. Therefore, when studying the $z$-motion, we fix the values of $x, P_{x}, \tau$ and $h$. Therefore, the
Hamiltonian of the $z$-motion,

$$
h_{z}=\frac{1}{2}\left[P_{z}^{2}+\left(x^{\prime}-\frac{z^{2}}{2}\right)^{2}\right]
$$

is the same for MFR and XO and coincides with the Hamiltonian considered by Zelenyi et al. (1990); Burkhart et al. (1991).

Characteristic phase portraits of the $z$-motion are shown in Fig. 2a for $x^{\prime}>0$ and in Fig. 2b for $x^{\prime}<0$. The separatrix $S_{z}$ in Fig. 2a is specified by $h_{z}=h_{z, s e p}=\left(x^{\prime}\right)^{2} / 2$. The action variable,

$$
I_{z}=\frac{1}{2 \pi} \oint P_{z} d z
$$

is equal to the normalized by $2 \pi$ area inside a phase curve on the $\left(z, P_{z}\right)$ plane. The value of $I_{z}$ is given by Sonnerup (1971); Büchner and Zelenyi (1989); Burkhart et al. (1991):

$$
I_{z}=\frac{8}{3 \pi} I_{z}^{\prime}=\frac{8}{3 \pi}\left(2 h_{z}\right)^{3 / 4} f(k)
$$

$f(k)=\left\{\begin{array}{cc}f_{a}(k)=\left(1-k^{2}\right) K(k)+\left(2 k^{2}-1\right) E(k), & k<1, \\ f_{b}(k)=\frac{1}{2}\left(2\left(1-k^{2}\right) k K\left(k^{-1}\right)+\left(2 k^{2}-1\right) k E\left(k^{-1}\right)\right), & k>1 .\end{array}\right.$

In (12), the subscript $a$ corresponds to the motion in the domain with $h_{z}>h_{z, s e p}$, and the subscript $b$ corresponds to the motion in one of two domains with $h_{z}<h_{z, s e p}$ (in what follows, we also use the notation $a$ and $b$ to denote the respective region);

$$
k^{2}=\frac{1}{2}\left(1+x^{\prime}\left(2 h_{z}\right)^{-1 / 2}\right)
$$

$E(k)$ and $K(k)$ are complete elliptic integrals. It is convenient to use $I_{z}^{\prime}$, which is just a constant multiple of $I_{z}$, in order to avoid repeating appearances of the factor $8 / 3 \pi$. 

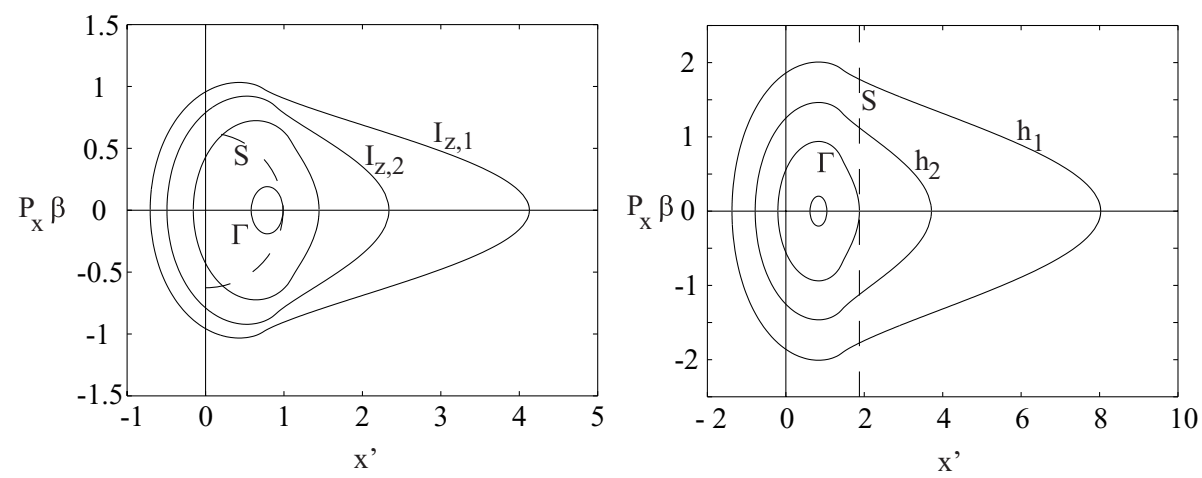

Fig. 3. Phase portraits on the $\left(x^{\prime}, P_{x} \beta\right)$ plane for the MFR problem: (a) $h=h_{0}$, phase curves are lines of constant $I_{z}$, the UC is a semi-circle $\left(x^{\prime}\right)^{2}+\left(P_{x} \beta\right)^{2}=2 h, x^{\prime}>0$, of two marked trajectories $h_{1}>h_{2}$; (b) $I_{z}=I_{z, 0}$, phase curves are lines of constant $h$, the $\mathbf{U C}$ is a straight line $x^{\prime}=\left(I_{z}^{\prime}\right)^{2 / 3}$, of two marked trajectories $I_{z, 1}<I_{z, 2}$.

\section{The motion on the $\left(x, P_{x}\right)$ plane.}

The action variable $I_{z}$ is the adiabatic invariant of the $x$ motion. Substituting $h_{z}=h_{z}\left(I_{z}, x^{\prime}\right)$ into (8) or (10), in other words, averaging the equations of motion over $z$-oscillations, we obtain the Hamiltonian of the $x$-motion possessing $1 \frac{1}{2}$ degrees of freedom:

$h=\frac{1}{2} P_{x}^{2} \beta^{2}(v x)+h_{z}\left(I_{z}, x^{\prime}\right) \quad(M F R)$,

$h=\frac{1}{2} P_{x}^{2}+h_{z}\left(I_{z}, x^{\prime}\right) \quad(X O)$.

The slow time, $\tau$, appears in (13) via the dependence of $x^{\prime}$ on $\tau$. Note, that in (13) the function $h_{z}$ is the same for the MFR and XO problems. But as $x^{\prime}$ depends on $x$ and $\tau$ differently for two problems, properties of the $x$-motion are also different.

We start our description of the $x$-motion with the construction of phase portraits.

\subsection{Phase portraits for MFR.}

There are two ways to plot a phase portrait on the $\left(x^{\prime}, P_{x} \beta\right)$ plane. One can either set $h=h_{0}$ and plot phase curves as lines of constant $I_{z}$ (Fig. 3a), or set $I_{z}=I_{z, 0}$ and plot phase curves as lines of constant $h$ (Fig. 3b). The advantages of both of these methods will become quite clear later (see Sect. 5 below). Every point on the $\left(x^{\prime}, P_{x} \beta\right)$ plane corresponds to a unique closed phase curve on the $\left(z, P_{z}\right)$ phase plane.

Figure $3 \mathrm{a}$ is very similar to what is shown in Büchner and Zelenyi (1989). The difference is that in Fig. 3a the vertical axis is $P_{x} \beta$ instead of $P_{x}$. The very similarity proves that by appropriate transformation of variables the problem with varying $B_{n}(x)$ can be reduced on some time scale to that of Büchner and Zelenyi (1989). This fact may not be immediately obvious from the original Hamiltonian structure (5).

The special curve $S$ (an uncertainty curve, UC, see Wisdom, 1985) corresponds to the separatrix of the $z$-motion.
When a phase point moving along a phase curve on the $\left(x^{\prime}, P_{x} \beta\right)$ plane arrives at $S$, the corresponding phase curve of the $z$-motion resides on $S_{z}$. In Fig. 3a the $\mathbf{U C}$ has the form of a semi-circle:

$$
\left(x^{\prime}\right)^{2}+\left(P_{x} \beta\right)^{2}=2 h, \quad x^{\prime}>0,
$$

and it is a straight line in Fig. 3b:

$$
x^{\prime}=\left(I_{z}^{\prime}\right)^{2 / 3} .
$$

The fixed point $C$ is the point of maximum of $f(k)$. At the maximum $k=k_{c} \approx 0.91$ and $f\left(k_{c}\right)=f_{c} \approx 1.16$. Hence

$$
\begin{gathered}
x_{c}^{\prime}=(2 h)^{-1 / 2}\left(2 k_{c}^{2}-1\right) \approx 0.47 h^{-1 / 2} \text { in Fig. 3a, } \\
x_{c}^{\prime}=\left(I_{z}^{\prime} / f_{c}\right)^{2 / 3}\left(2 k_{c}^{2}-1\right) \approx 0.59\left(I_{z}^{\prime}\right)^{2 / 3} \text { in Fig. 3b. }
\end{gathered}
$$

In Fig. 3a the outer orbits correspond to the smaller values of $I_{z}^{\prime}$, while the inner orbits correspond to the larger values of $I_{z}^{\prime}$, with $I_{z}^{\prime}$ reaching its maximum value of $I_{z, \max }^{\prime}=f_{c}(2 h)^{3 / 4}$ at $C$. Similarly, in Fig. $3 \mathrm{~b}$ the outer orbits correspond to the larger values of $h$, while the inner orbits correspond to the smaller values of $h$. On both phase portraits there is a unique curve $\Gamma$ that is tangent to $S$. It divides the phase plane into two regions: the region $A$ outside $\Gamma$ and the region $B$ within $\Gamma$. All the trajectories residing in the region $B$ do not cross $S$, and all the trajectories residing in the region $A$ cross $S$.

\subsection{Phase portraits in the vicinity of an $X$-line}

As Hamiltonian (6) depends on the parameter $s$, the phase portraits of the $x$-motion in the vicinities of neutral lines of $X$ - and $O$-types are significantly different. The behaviour of particles in the vicinity of an $X$-line was studied in several papers (see e.g. Martin, 1986; Burkhart et al., 1991). In Burkhart et al. (1991) the phase portraits were numerically constructed as surface of sections of trajectories of the original system. Contrarly to that, the phase portraits presented below are constructed purely analytically using the method 

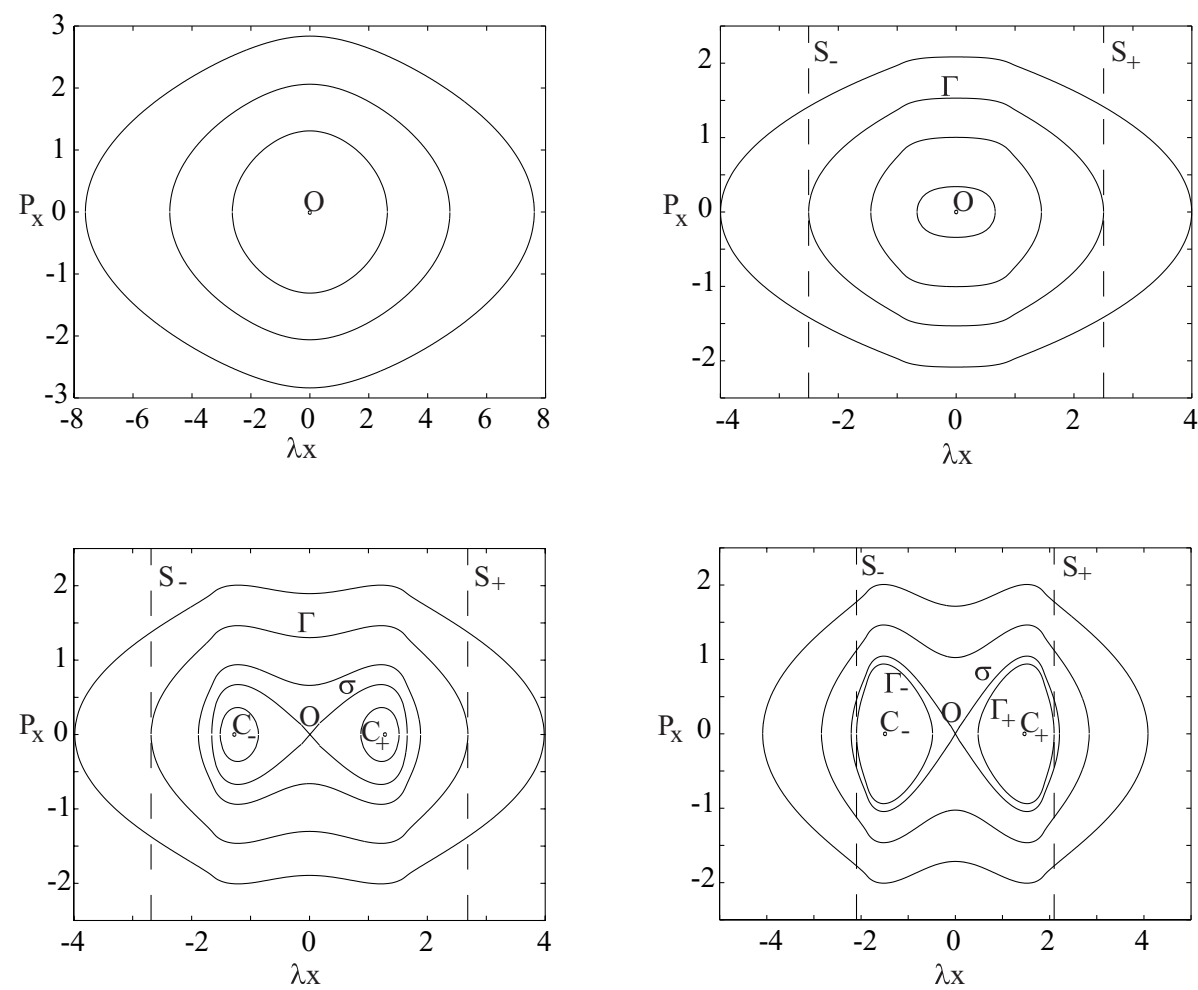

Fig. 4. Phase portraits on the $\left(\lambda x^{\prime}, P_{x}\right)$ plane for the $X$-line problem, $I_{z}=I_{z, 0}$, phase curves are lines of constant $h$. (a) $\tau<-\left(I_{z}^{\prime}\right)^{2 / 3}$, (b) $-\left(I_{z}^{\prime}\right)^{2 / 3}<\tau<\tau_{c}$, (c) $\tau_{c}<\tau<0$, (d) $\tau>0$.

of averaging and the perturbation theory. As a result, we were able to describe the structure of phase portraits of $\mathrm{x}$ motion in more details. In particular, we found that there were not three, but four qualitatively different types of the phase portraits depending on the values of the slow variables and $I_{z}^{\prime}$ (see below).

We construct phase portraits for the $X O$ problem in the same way the phase portrait in Fig. $3 \mathrm{~b}$ was constructed: on the whole phase plane $I_{z}=I_{z, 0}$ and the phase curves are lines of constant $h$.

Depending on the value of $\tau$, there are four qualitatively different types of phase portraits for the particles residing near an $X$-line.

1). $-\infty<\tau<-\left(I_{z}^{\prime}\right)^{2 / 3}$

A typical phase portrait is shown in Fig. 4a. For these values of $\tau$, there is no $\mathbf{U C}$. Particles do not cross the equatorial $(z=0)$ plane. Hence, they reside within the region $B$ on the $\left(z, P_{z}\right)$ phase plane. The only stationary point is at the origin.

2). $-\left(I_{z}^{\prime}\right)^{2 / 3}<\tau<\tau_{c}$

A typical phase portrait is shown in Fig. 4b. The critical value $\tau_{c}$ is

$$
\tau_{c}=-x_{c}^{\prime}
$$

where $x_{c}^{\prime}$ is given by (15). The $\mathbf{U C}$ consists of two straight lines, $S_{ \pm}$, that are parallel to the $P_{x}$-axis and are separated from it by a distance

$$
(\lambda x)_{s}=\sqrt{2\left(-\tau-\left(I_{z}^{\prime}\right)^{2 / 3}\right)} .
$$

The only stationary point is still at the origin. The phase curve $\Gamma$, that is tangent to $\mathbf{U C}$, divides the phase plane into two regions: $A$ and $B$.

3). $\tau_{c}<\tau<0$.

A typical phase portrait is shown in Fig. 4c. At $\tau=\tau_{c}$ the elliptic stationary point $O$ at the origin undergoes a bifurcation: the point $O$ becomes hyperbolic and there are two new elliptic stationary points, $C_{ \pm}$, located at

$$
(\lambda x)_{c}= \pm \sqrt{2\left(\tau-\tau_{c}\right)}
$$

There is a new special curve on the phase portrait - the separatrix of the $x$-motion, $\sigma$. The curve $\sigma$ separates the trajectories that are localized on one side of the $X$-line (those inside $\sigma$ ) from those that move from side to side of the $X$ line within one period of the $x$-motion.

4). $\tau>0$. 
A typical phase portrait is shown in Fig. 4 d. At $\tau=0, \sigma$ is tangent to $S_{ \pm}$and for larger values of $\tau$, crosses it. The curve $\Gamma$ now consists of two separate curves $\Gamma_{ \pm}$and, therefore, the region $B$ consists of two separate domains, $B_{+}$and $B_{-}$, that are bounded by $\Gamma_{+}$and $\Gamma_{-}$, respectively.

\subsection{Phase portraits in the vicinity of an $O$-line.}

Depending on the value of $\tau$ there are three qualitatively different types of phase portraits.

$$
\text { 1). }-\infty<\tau<-\left(I_{z}^{\prime}\right)^{2 / 3} \text {. }
$$

A typical phase portrait is shown in Fig. 5a. The UC has the form of two straight lines $S_{ \pm}$, that are parallel to the $P_{x}$ axis and are separated from it by a distance

$$
(\lambda x)_{s}=\sqrt{2\left(-\tau-\left(I_{z}^{\prime}\right)^{2 / 3}\right)} .
$$

There are three fixed points: $O$ (hyperbolic) at the origin and $C_{ \pm}$(elliptic) at

$$
(\lambda x)_{c}= \pm \sqrt{2\left(-\tau+\tau_{c}\right)} .
$$

2). $-\left(I_{z}^{\prime}\right)^{2 / 3}<\tau<\tau_{c}$.

A typical phase portrait is shown in Fig. 5b. For these values of $\tau$, there is no $\mathbf{U C}$, hence, the motion of particles on the entire phase plane is regular outside of a small domain in the vicinity of $\sigma$.

3). $\tau_{c}<\tau$.

A typical phase portrait is shown in Fig. 5c. At $\tau=\tau_{c}$ the hyperbolic fixed point $O$ and the elliptic fixed points $C_{ \pm}$ merge giving rise to a single elliptic fixed point at the origin.

\subsection{Motion in the region $B$}

In both (MFR and XO) problems the behaviour of particles residing in the regions $A$ and $B$ is drastically different.

In the region $B$, both in MFR and XO problems, the phase space is filled by the invariant tori up to a residue of exponentially small measure (of order of $\sim \exp (-C / \sqrt{\kappa})$ where $C$ is some constant) and the value of adiabatic invariant, $I_{z}$, is conserved eternally: on infinite time interval it has only oscillations of order $\kappa$ (the Arnold theorem about perpetual adiabatic invariance, see e.g. Arnold et al., 1988). We can introduce the second action variable

$$
I_{x}=\frac{1}{2 \pi} \oint P_{x} d x,
$$

where the integral is taken over a closed phase trajectory on the $\left(x, P_{x}\right)$ plane.

\subsection{Motion in the region $A$}

In the region $A$, the motion is more complicated. The value of $I_{z}$ is conserved only far from $S$ and undergoes a jump every time a phase point crosses $S$. The asymptotic formulas for the jump of adiabatic invariant on a separatrix were obtained by Timofeev (1978) for particular case of a pendulum in a slowly varying gravity field, by Neishtadt (1986) and Cary et al. (1986) for systems with one degree of freedom plus slowly varying parameter and by Neishtadt (1987) for systems with two degrees of freedom, one corresponding to the fast motion and the other corresponding to the slow motion. This theory was first applied to magnetospheric problems by Büchner and Zelenyi (1989). We have

$$
\begin{aligned}
\Delta I_{z}=I_{z, a}-2 I_{z, b} & \approx \mp \frac{4}{\pi} \kappa \beta^{2} P_{x} \ln (2 \sin \Theta) \quad(\text { MFR }), \\
& \approx \mp \frac{1}{\pi} \lambda(\lambda x) P_{x} \ln (2 \sin \Theta) \quad(X O) .
\end{aligned}
$$

In (18), $I_{z, a}$ and $I_{z, b}$ are the values of $I_{z}$ in the domains $a$ and $b$, respectively, the minus and plus signs correspond to passages from $b$ to $a$ and from $a$ to $b$, respectively (see the discussion below Eq. (12) in Sect. 3). The phase $\Theta$ characterizes a separatrix crossing and it depends not only on $x$ and $P_{x}$ but on $z$ and $P_{z}$ as well. The value of $\Theta$ is very sensitive to small perturbations of the initial conditions and can be treated as a random variable uniformly distributed on $(0, \pi)$. It is shown by Neishtadt (1987), that in the limit $\kappa \rightarrow 0$, for multiple crossings $\Delta I_{z}$ can be treated as a random value with a zero mean and dispersion of order $\sim \kappa^{2}$ (MFR) or (due to normalizing conditions $\lambda x \sim 1$ ) of order $\sim \lambda^{2}$ (XO). Because of these jumps, $I_{z}$ was referred to as a quasi-adiabatic invariant by Büchner and Zelenyi (1989).

Because of the jumps in $I_{z}$ no unique value of $I_{z}$ can be assigned to a whole orbit of the $x$-motion. (Moreover, in the exact system projections of phase curves on the $\left(x, P_{x}\right)$ phase plane are not even closed curves - while in the averaged system the trajectories are closed.) Nevertheless, while a particle moves far from the uncertainty curve the value of $I_{z}$ remains nearly constant (with accuracy of order $\kappa$, see the discussion in the previous subsection) and can be used to specify segments of trajectories between consecutive crossings of the uncertainty curve. Recall, that the characteristic values of the jumps' magnitude are small (of order $\sim \kappa$ ). Therefore, at every single crossing the value of $I_{z}$ does not change much. It is the accumulation of the jumps (see Sect. 5 below) that leads to chaotic dynamics and mixing in the phase space. Despite the jumps, the notion of $I_{z}$ was used to characterize orbits by many different authors (see e.g. Karimabadi et al., 1990; Burkhart et al., 1991; Moses et al., 1993). In particular, the value of $I_{z}$ characterizes the motion in a sense that it foretells if a given trajectory (for frozen values of the slow variables) intersects the separatrix or not. There exists a critical value of $I_{z}$ (see Büchner and Zelenyi, 1989, and Subsects. 4.14.3) such that if at any point along a trajectory $I_{z}$ is larger (smaller) then that critical value, the trajectory does (does 

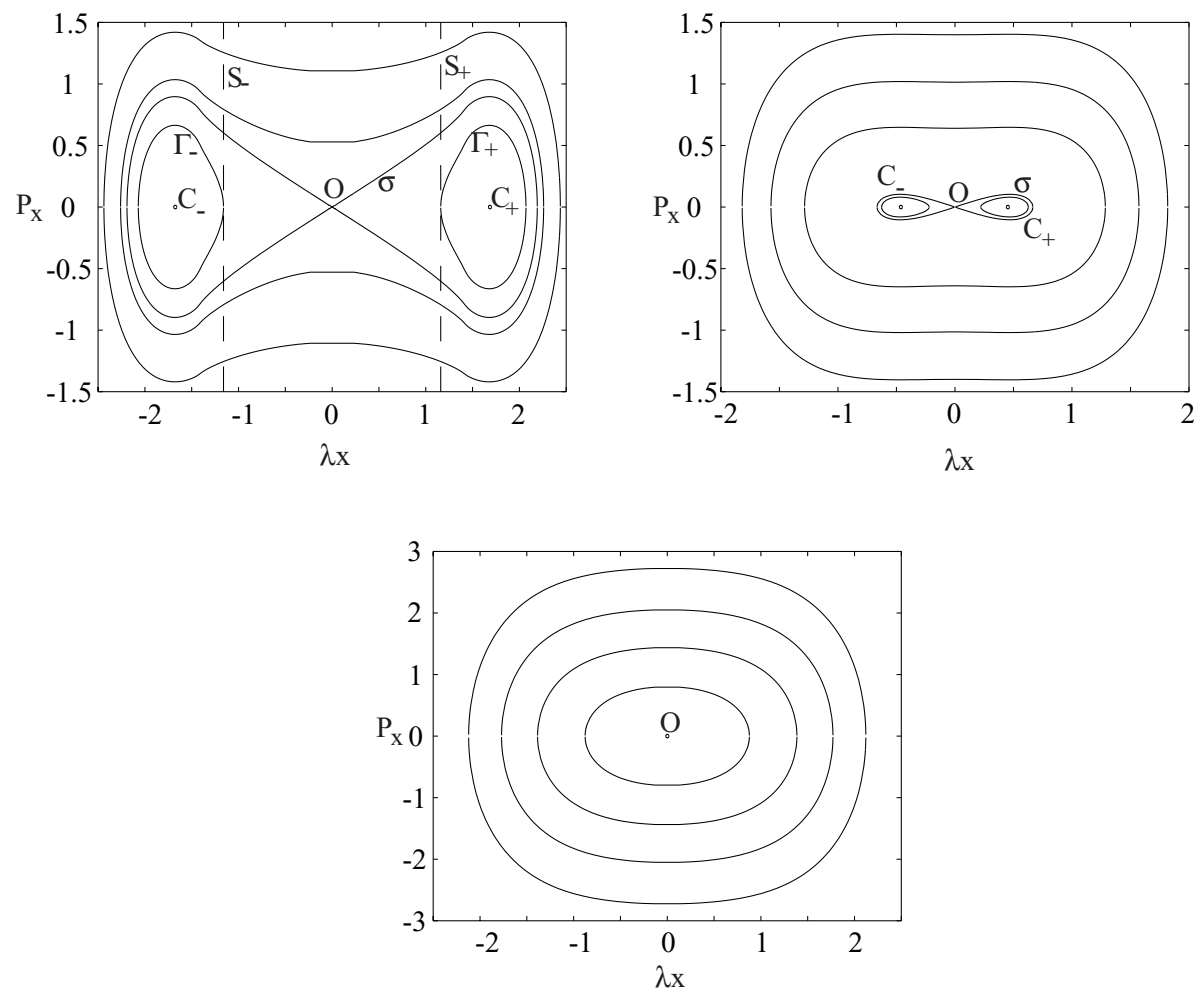

Fig. 5. Phase portraits on the $\left(\lambda x^{\prime}, P_{x}\right)$ plane for the $O$-line problem, $I_{z}=I_{z, 0}$, phase curves are lines of constant $h$. (a) $\tau<-\left(I_{z}^{\prime}\right)^{2 / 3},(\mathbf{b})$ $-\left(I_{z}^{\prime}\right)^{2 / 3}<\tau<\tau_{c},(\mathbf{c}) \tau>\tau_{c}$.

not) intersect the separatrix. Jumps of $I_{z}$ can not move $I_{z}$ across the critical value. Besides that, the current value of $I_{z}$ such important properties of the $x$-motion as the period and the distance of the closest approach to the earth (the location of the rightmost point in Fig. 3).

Despite the jumps of $I_{z}$ in the region $A$, we can formally introduce the action $I_{x}$ as the normalized by $2 \pi$ area within a curve $h=$ const, $I_{z}=$ const on the $\left(x, P_{x}\right)$ plane. $I_{x}$ undergoes jumps, that are synchronized with the jumps of $I_{z}$ :

$$
\Delta I_{x} \sim \frac{\partial I_{x}}{\partial I_{z}} \Delta I_{z}
$$

As the jumps of $I_{z}$ are small, $\Delta I_{z} \sim \kappa$, the corresponding jumps of $I_{x}$ are also small. Hence, although even the original adiabatic invariant, $I_{z}$, is not exactly conserved, we can introduce and use the second adiabatic invariant in the case when the aggregate change in $I_{z}$ is small (see Sect. 5 for more details). Therefore, $I_{x}$ can also be called a quasi-adiabatic invariant and plays the role of the longitudinal invariant in GCT.

For the XO problem we can obtain the explicit formula for $I_{x}$, that is valid in the region $A$ as well as in the region $B$. The similar formula for the MFR problem for $B_{n}=$ const was derived by Zelenyi et al. (1990). It follows from (12) and (17), that
$I_{x}=\alpha \frac{2 \sqrt{2}}{\pi} I_{z}^{\prime} \int_{k_{1}}^{k_{2}} \frac{Q(k)}{f^{2}(k)}\left[\frac{2 h\left(I_{z}^{\prime}\right)^{-4 / 3} f^{4 / 3}(k)-1}{\left(2 k^{2}-1\right)+\tau\left(I_{z}^{\prime}\right)^{-2 / 3} f^{2 / 3}(k)}\right]^{1 / 2} d k$,

where

$$
Q(k)=\left\{\begin{array}{cc}
k K(k), & k<1, \\
K\left(k^{-1}\right), & k>1
\end{array}\right.
$$

and $f(k)$ was defined in (12). The values of $k_{1}, k_{2}$ and $\alpha$ depend on whether a particle moves in the vicinity of $X$ - or $O$-line and on the position of a phase curve on the phase plane.

For particles in the vicinity of an $X$-line $k_{2}$ is given by the conditions $k_{2}>k_{c}$ and

$$
2 h f^{4 / 3}\left(k_{2}\right)=\left(I_{z}^{\prime}\right)^{4 / 3} .
$$

The values of $k_{1}$ and $\alpha$ depend on whether a phase curve intersects the line $x=0$ or not. If the whole curve is on one side of line $x=0$ then $k_{1}$ is given by (20) and the condition $k_{1}<k_{c}$; in this case $\alpha=1$. If a phase curve intersects line the $x=0$ then $\alpha=2$ and $k_{1}$ is given by

$$
-\tau=\left(I_{z}^{\prime} / f\left(k_{1}\right)\right)^{2 / 3}\left(2 k_{1}^{2}-1\right) .
$$


For a particle in the vicinity of an $O$-line $k_{1}$ is given by the conditions $k_{1}<k_{c}$ and

$$
2 h f^{4 / 3}\left(k_{1}\right)=\left(I_{z}^{\prime}\right)^{4 / 3}
$$

If the whole curve is on one side of the line $x=0$ then $k_{2}$ is given by (21) and the condition $k_{2}>k_{c}$; in this case $\alpha=1$. If a phase curve intersects line $x=0$ then $\alpha=2$ and $k_{2}$ is given by

$$
-\tau=\left(I_{z}^{\prime} / f\left(k_{2}\right)\right)^{2 / 3}\left(2 k_{2}^{2}-1\right) .
$$

\section{Long-time dynamics: regular transport and mixing}

The earth magnetotail is an open system. Charged particles from the solar wind enter in the far region and then accelerate and drift towards the earth. In the present section we discuss the properties of particles' dynamics on the time scales of the life time of particles in the magnetotail, which for a typical particle is much longer then the period of the $x$-motion.

After averaging over the $x$-motion, we can study the motion on the $(\tau, h)$ plane (i.e. the acceleration of the particles). For given $I_{z}$ and $I_{x}$, every point on the $(\tau, h)$ plane corresponds to the entire phase curve on the $\left(x, P_{x}\right)$ plane.

In the absence of the electric field the particles would stay in the region where they originally resided $(A$ or $B)$ forever. As it was discussed before, the jumps of adiabatic invariants cannot move particles from the region $A$ to the region $B$ (recall, the particle in the region $B$ do not cross the separatrix). It is the presence of the electric field combined with the longitudinal variations of the magnetic field that causes the energy drift and the particles move from the region $B$ to the region $A$, or from the region $A$ to the region $B$. The direction of the drift depends on the location of a particle in the magnetotail.

\subsection{Region $B$}

While a particle resides in the region $B$, the values of both adiabatic invariants, $I_{z}$ and $I_{x}$, are conserved up to the oscillations of order $\kappa$ (see Subsect. 4.4). The motion of a particle on the $(\tau, h)$ plane is described by conservation laws

$$
I_{z}=\text { const }, \quad I_{x}=\text { const. }
$$

Let us consider the drift in energy for the MFR problem. On the $\left(x^{\prime}, P_{x} \beta\right)$ phase plane in Fig. 3b, the shape of the phase curves does not change with time, each trajectory being specified by a value of energy. Consequently, as the energy drifts, particle drift from one phase curve to another. The direction of this drift depends on the sign of $d h / d t$ averaged over the $x$-motion:

$$
\frac{d h}{d t}=\frac{\partial h}{\partial t}=\varepsilon v\left\langle P_{x}^{2} \beta \frac{\partial \beta}{\partial(\nu x)}\right\rangle,
$$

where brackets denote the averaging over the $x$-motion. It follows from (23), that the particle's acceleration is affected only by large-scale variations of $\beta(v x)$ with a typical length scale comparable with the amplitude of $x$-oscillations.
The experimental observations show (see Tsyganenko (1987)) that, on average, $\partial \beta / \partial x>0$ (i.e. $B_{n}(x)$ increases towards the earth). Hence, the energy increases with time and particles move from the region $B$ to the region $A$. The time $t_{\Gamma}$ that a particle spends in the region $B$ (in other words, the time before a particle reaches $\Gamma$ in Fig. 3b) is given by

$$
I_{x}\left(h_{0}, t_{0}\right)=I_{x}\left(h_{\Gamma}, t_{\Gamma}\right),
$$

where $h_{0}$ is the initial energy of the particle and

$$
h_{\Gamma}=\frac{1}{2}\left(I_{z}^{\prime}\right)^{4 / 3}
$$

is the energy corresponding to $\Gamma$.

\subsection{Region $A$}

The motion of particles in the region $A$ is affected by two phenomena: (1) the drift over energy under the influence of the electric field and (2) the jumps of adiabatic invariants. The behavior of particles depends on the relative importance of these phenomena.

Wiggins (1988) proved the presence of Smale horseshoes in domains with separatrix crossings, which, in turn, implies the existence of chaotic trajectories. The accumulation of the jumps of adiabatic invariants could produce chaotic dynamics, a phenomena called adiabatic chaos in Wiggins (1988). For the problem under consideration the presence of chaotic dynamics was demonstrated in (Büchner and Zelenyi, 1989; Zelenyi et al., 1990). The properties of the long-term evolution of adiabatic invariants depend on whether consecutive crossings are statistically dependent or independent.

In general case, the statistical independence follows from the divergence of phases along trajectories, which, in turn, depends on the relation between the values of the phase $\Theta$ (see Eq. 18) at two successive separatrix crossings. Denote the respective values of $\Theta$ as $\Theta_{1}$ and $\Theta_{2}$. A small variation $\delta \Theta_{1}$ of $\Theta_{1}$ produces the variation of the jump value of $I_{z}$ by a quantity of order $\kappa \delta \Theta_{1}$. As a result, the phase $\Theta_{2}$ changes by the quantity $\delta \Theta_{2} \sim C(1 / \kappa) \kappa \delta \Theta_{1}=C \delta \Theta_{1}$. The coefficient $C$, that is proportional to $\partial T_{x} / \partial I_{z}$, defines the divergence of trajectories. If $C$ is large trajectories diverge fast: a small change in the resonance phase before one crossing generally results in a big change in the value of the resonance phase of the next crossing. In that case the jumps of adiabatic invariant at two successive crossings can be considered as independent. On the other hand, small values of $C$ lead to nearly adiabatic motion.

With consecutive jumps being independent, evolution of the adiabatic invariant due to multiple separatrix crossings can be treated as a random walk with a step of order of $\kappa$ without a preferred direction. Such a diffusion of adiabatic invariants is observed in (nearly all) numeric simulations of various systems. Diffusion time can be estimated as a characteristic slow period divided by a characteristic mean square value of the jump. Thus estimated, diffusion time was shown to be in a good agreement with numerical simulations (see Bruhwiler and Cary, 1989). 
The assumption of statistical independence is crucial for chaotization: when phases on the successive crossings are related a regular motion can emerge. Cary and Skodje (1989) showed that for some initial conditions consecutive crossings are statistically dependent. It was shown by Elskens and Escande (1991) and Neishtadt et al. (1997) that the islands of stability, albeit being of a small measure, do exist inside large chaotic sea. A similar phenomenon of statistical dependence of consecutive jumps in a model of the roll convection was discussed in Itin et al. (2002). A somewhat different phenomenon appears if $T_{x}$ is (approximately) independent of $I_{z}$. In this case $\partial T_{x} / \partial I_{z}$ approximately vanishes and consecutive phases become correlated. In particular, consecutive jumps may cancel each other producing a phenomenon called energy resonances or beamlets (see e.g. Chen, 1992; AshourAbdalla et al., 1993), that is well known in magnetospheric applications, but involves only a relatively small number of particles in the process of the convection from the distant tail to the earth. For nearby trajectories the net effect of jumps (averaged for all the crossings during one period of the $x$-motion), although not exactly vanishing, is much smaller than in a general case. Consequently, the beamlet particles behave as if they are in an adiabatic regime. It is important to note that the beamlets are occur only for not too small values of $\kappa$. In the formal limit of $\kappa \rightarrow 0$ the beamlets disappear. We return to the topic of the beamlets in the end of the current section where we discuss the rate of mixing.

In the pure parabolic field model the particles stay in the magnetotail forever. In that case, the mixing is complete and all the structures in the distribution functions of the chaotic region that were present when particles entered the magnetotail are washed out. On the other hand, in real magnespheric configurations the particles spend in the magnetotail only a finite time, and the number of jumps may not be sufficient for complete mixing.

Introduce the characteristic time of mixing, $T_{j}$, as a time over which the value of the adiabatic invariant changes by a quantity of order 1 (see below). As the jumps of the adiabatic invariant are localized near the uncertainty curve, $\mathbf{U C}, T_{j}$ can be written as

$$
T_{j} \sim T_{p e r} \frac{1}{D},
$$

where $T_{\text {per }}$ is a typical time between consecutive crossings of $\mathbf{U C}$ and is of order of the period of the $x$-motion. $D$ is the diffusion coefficient and is of order of the average square of the magnitude of a jump of the adiabatic invariant at one crossing:

$$
D \sim\left\langle\left(\Delta I_{z}\right)^{2}\right\rangle
$$

The extent of mixing in the system depends on the relation between $T_{j}$ and $T_{L}$, that is a characteristic lifetime of particles in the magnetotail. If $T_{j}$ is larger then $T_{L}$, then mixing is small everywhere in the phase space and distribution functions are transported towards the earth relatively intact. Contrarily, if $T_{j}$ is less then $T_{L}$, then mixing is important and distribution functions are homogenized.
Introduce a parameter

$$
\eta=\frac{T_{L}}{T_{j}} .
$$

In MFR, the value of $T_{p e r}$ is of order of $\sim 1 / \kappa$. A characteristic size of jumps of the adiabatic invariant on the separatrix is of order $\sim \kappa$. Therefore, $T_{j} \sim \kappa^{-3}$.

The lifetime of particles is defined by the acceleration due to the electric field and depends on the magnitude of the constant solar wind-induced electric field and non-uniformity of magnetic field. A typical rate of change of energy is of order of $\varepsilon v$ (see Eq. 23). Hence, we have $T_{L} \sim(\varepsilon v)^{-1}$ and we arrive at

$$
\eta=\frac{\kappa^{3}}{\nu \varepsilon}
$$

Similarly, in XO $T_{L} \sim \varepsilon^{-1}, T_{j} \sim \lambda^{-3}$ and

$$
\eta=\frac{\lambda^{3}}{\varepsilon} .
$$

If $\eta \ll 1$, drift dominates, jumps are small perturbations and the mixing is negligible. In the opposite case, $\eta \gg 1$, the mixing is strong: particles cross the separatrix sufficient number of times for adiabatic invariant cover the whole admissible domain. A similar observation was made (based on numerical simulations) by Karimabadi et al. (1990). Therefore, $\eta$ is a measure of mixing in the system.

Now one can see the advantages of the two types of phase portraits on the $\left(x^{\prime}, P_{x}\right)$ plane. If $\eta \ll 1, I_{z}$ remains approximately constant and, like in the region $B$, particles drift from one phase curve to another in Fig. 3a. On the other hand, if $\eta \gg 1$, particles jump from one phase curve to another in Fig. 3b remaining on approximately the same energy level.

Note, that if $D$ is defined by (24), $\eta$ is an averaged characteristics, that is the same for all the phase space. In order to describe the evolution of coherent structures, we can consider $\eta$ as a function of (say) the energy $h$. The most prominent consequence of the dependence of $\eta$ on $h$ is a possibility for consecutive jumps to be correlated. To include the correlation in the diffusion coefficient we can redefine $D$ based on the average square of the magnitude of a total jump of the adiabatic invariant in one period of the $x$-motion:

$$
D(h) \sim\left\langle\left(\Delta_{2} I_{z}\right)^{2}\right\rangle / 2
$$

If the consecutive crossings are statistically independent, $\Delta_{2} I_{z}$, that is a sum of two changes of $I_{z}$ during one period of the $x$-motion, is of order of the variation of $\Delta I_{z}$ at one crossing. However, if for a certain value of $h=h_{b}$ the jumps are correlated, like in the case of beamlets, when they cancel each other, $\Delta_{2} I_{z}$ (and, correspondingly, $D\left(h_{b}\right)$ ) may go all the way to zero. In this case $\eta$ goes to 0 and at the energy level $h=h_{b}$ the dynamics is adiabatic. 


\section{Orbits with small $I_{z}$ in MFR}

If the cumulative effect of the jumps of the adiabatic invariant is small $(\eta \ll 1)$, then the mixing is weak in the whole domain and Eq. (22) specify the acceleration rate. In the general case, the exact solutions cannot be obtained explicitly.

These solutions can be simplified in a particular case $I_{z} \ll 1$, that corresponds to very long orbits with far reflecting points (a reflecting point being the rightmost point on a phase curve in Fig. 3a, see also Sect. 4). However, one must keep in mind that the separation of the $x$ - and $z$-motions is valid only in the region where $\beta \sim 1$ and, therefore, we can not use the considerations below to describe the motion of the particles that come too close to the earth.

For large $x^{\prime}$ (see 9) we can linearize Hamiltonian (8) near $z_{0}= \pm \sqrt{2 x^{\prime}}$. Introducing $z=z_{0}+\bar{z}$, where $\bar{z} \ll z_{0}$, we get

$$
h=\frac{1}{2}\left(P_{x}^{2} \beta^{2}+P_{z}^{2}+2 x^{\prime} \bar{z}^{2}\right)=\frac{1}{2} P_{x}^{2} \beta^{2}+h_{z} .
$$

The result is a harmonic oscillator with a variable frequency $\omega\left(x^{\prime}\right)=\sqrt{2 x^{\prime}}$ and, therefore,

$$
h_{z}=\frac{1}{2}\left(P_{z}^{2}+\bar{z}^{2} \omega^{2}\left(x^{\prime}\right)\right)=I_{z} \omega\left(x^{\prime}\right) .
$$

It follows from (17) and (25), that

$$
I_{x} \approx \frac{1}{\pi} \int_{x_{\min }^{\prime}}^{0} P_{x} d x+\int_{0}^{x_{\max }^{\prime}} \frac{1}{\beta\left(x^{\prime}\right)} \frac{1}{\kappa} \sqrt{2 h-I_{z} \sqrt{8 x^{\prime}}} d x^{\prime},
$$

where $x_{\min }^{\prime}$ and $x_{\max }^{\prime}$ are given by the condition $P_{x}\left(x=x_{\min , \max }\right)=0$. One can see in Fig. 3a that the first term in (26) is much smaller then the second one and thus can be neglected. Introduce a new variable

$$
\xi=\sqrt{2 x^{\prime}} I_{z} / h .
$$

In terms of $\xi$, (26) can be written as

$$
I_{x} \approx \frac{\sqrt{2}}{\pi} \frac{h^{5 / 2}}{\kappa I_{z}^{2}} \int_{0}^{1} \frac{1}{\beta} \xi \sqrt{1-\xi} d \xi .
$$

In (27) we substituted $\xi_{\max }=1$. Integrating (27) by parts and taking into account that

$$
\frac{d \beta}{d \xi}=\frac{1}{\kappa} \frac{d \beta}{d x} \frac{h^{2}}{I_{z}^{2}} \xi \quad \text { and } \quad \frac{d \beta}{d x} \frac{1}{\beta} \sim v .
$$

we get

$I_{x} \approx \frac{2}{3} \frac{\sqrt{2}}{\pi} \frac{h^{5 / 2}}{\kappa I_{z}^{2}}\left[\frac{2}{5} \frac{1}{\beta}-\frac{\nu}{\kappa} \frac{h^{2}}{I_{z}^{2}} \int_{0}^{1}\left((1-\xi)^{3 / 2}-\frac{3}{5}(1-\xi)^{5 / 2}\right) \frac{1}{\beta} d \xi\right]$.

The first term in the square brackets in (28) depends on the average over an $x$-oscillation value of $\beta$. The second term describes variations of $\beta$ over an $x$-orbit. The relative importance of the second term in (28) is given by a parameter

$$
\gamma=\frac{5}{2} \frac{v}{\kappa} \frac{h^{2}}{I_{z}^{2}} .
$$

If $\gamma \ll 1$ the second term is small and

$$
I_{x} \approx \frac{4 \sqrt{2}}{15 \pi} \frac{h^{5 / 2}}{\kappa I_{z}^{2}} \frac{1}{\beta} .
$$

Using (22) we obtain the acceleration rate,

$$
h \sim \beta^{2 / 5},
$$

that confirms the result obtained by Zelenyi et al. (1990). Experimental results yield that the condition $\gamma \ll 1$ is satisfied for the particles with $I_{z} \ll 1$ beyond $50 R_{E}$ from the Earth. Note, that if $\gamma \geq 1,(29)$ is not valid.

The period of $x$-oscillations is given by

$$
T_{x}=2 \int_{x_{\min }}^{x_{\max }} \frac{d x}{\dot{x}}=2 \int_{x_{\min }}^{x_{\max }} \frac{d x}{P_{x} \beta^{2}} \approx \sqrt{2} \frac{h^{3 / 2}}{\kappa I_{z}^{2}} \int_{0}^{1} \frac{1}{\beta} \frac{\xi d \xi}{\sqrt{1-\xi}} .
$$

Recall that the mixing parameter, $\eta$, is proportional to the period of the $x$-motion, $T_{x}$. Taking into account that the characteristic values of $h$ are of order 1 , we get that for small $I_{z}$, $\eta$ has the form

$$
\eta \sim I_{z}^{2} \frac{\kappa^{3}}{\nu \varepsilon}
$$

It follows from (30), that for the particles with small $I_{z}$ the mixing is less prominent then for the particles with $I_{z} \sim 1$ as the particles with $I_{z} \ll 1$ cross the separatrix fewer times before being carried out of the magnetotail. The similar effect (that for shorter trajectories the rate of diffusion is larger) was observed by Karimabadi et al. (1990).

\section{Acceleration of particles in XO}

Again, consider the case $\eta \ll 1$ (small jumps of $I_{z}$ ). Conservation laws (22) are valid on the whole phase plane. The motion on the (time, energy) phase plane can be described using a single phase portrait. Apply a change of variables

$$
\begin{aligned}
x & =\bar{x}\left(I_{z}^{\prime}\right)^{1 / 3}, \quad h=\bar{h}\left(I_{z}^{\prime}\right)^{4 / 3}, \quad \tau=\bar{\tau}\left(I_{z}^{\prime}\right)^{2 / 3} . \\
p_{x} & =\bar{p}_{x}\left(I_{z}^{\prime}\right)^{-1 / 3}, h_{z}=\bar{h}_{z}\left(I_{z}^{\prime}\right)^{4 / 3},
\end{aligned}
$$

Note, that the value of $k$ does not change under such a change of variables. Introduce a new adiabatic invariant

$$
J=I_{x} / I_{z}^{\prime} .
$$

It follows from (19), that

$$
J=\alpha \frac{2 \sqrt{2}}{\pi} \int_{k_{1}}^{k_{2}} \frac{Q(k)}{f^{2}(k)}\left[s \frac{2 \bar{h} f^{4 / 3}(k)-1}{-\left(2 k^{2}-1\right)-\bar{\tau} f^{2 / 3}(k)}\right]^{1 / 2} d k .
$$



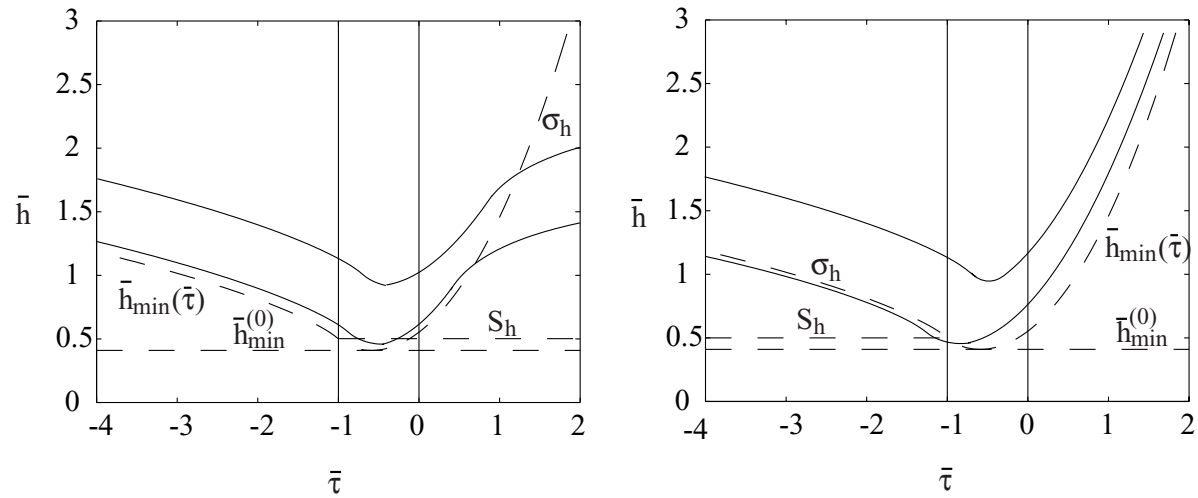

Fig. 6. Phase portraits on the $(\bar{\tau}, \bar{h})$ plane for (a) the $X$-line problem, (b) the $O$-line problem. Phase curves are lines of constant $J$.

On the $(\bar{\tau}, \bar{h})$ plane phase curves are lines of constant $J$; the shape of curves does not depend on $I_{z}$.

It follows from (31), that the lowest possible value of $h$ is $h_{\min }^{(0)}=\left(1 / f_{c}\right)^{4 / 3}$, although phase curves do not fill the entire domain $\bar{h}>\bar{h}_{\text {min }}^{(0)}$. The minimum possible energy $h_{\min }$ as a function of $\bar{\tau}$ is given by

$$
\bar{h}_{\min }=\left\{\begin{array}{c}
\bar{h}_{\min }(\bar{\tau}), \bar{\tau}<\bar{\tau}_{c}, \\
\bar{h}_{\min }^{(0)}, \quad \bar{\tau}>\bar{\tau}_{c},
\end{array}\right.
$$

for particles moving in the vicinity of an $X$-line and

$$
\bar{h}_{\min }=\left\{\begin{array}{c}
\bar{h}_{\min }^{(0)}, \quad \bar{\tau}<\bar{\tau}_{c}, \\
\bar{h}_{\min }(\tau), \bar{\tau}>\bar{\tau}_{c},
\end{array}\right.
$$

for particles moving in the vicinity of an $O$-line, where $\bar{h}_{\min }(\bar{\tau})$ and $\bar{\tau}_{c}$ (see Eqs. 15 and 16) are given by

$$
\begin{aligned}
& (2 \bar{h})^{4 / 3} f\left(k_{h \tau}\right)=1, \\
& 2 k_{h \tau}^{2}-1=-\bar{\tau}(2 \bar{h})^{-1 / 2}, \\
& \bar{\tau}_{c}=-\frac{1}{f_{c}^{2 / 3}}\left(2 k_{c}^{2}-1\right) \approx-0.59 .
\end{aligned}
$$

In the vicinity of an $X$-line, Eqs. (32) define for $\bar{\tau}>\bar{\tau}_{c}$ on the $(\bar{\tau}, \bar{h})$ plane curve $\sigma_{h}$, that corresponds to $\sigma$ on the $\left(x^{\prime}, P_{x}\right)$ plane. In the vicinity of an $O$-line, Eqs. (32) define $\sigma_{h}$ for $\bar{\tau}<\bar{\tau}_{c}$. In both cases, above $\sigma_{h}$ particles cross the $x=0$ plane twice within each period of the $x$-motion. Below $\sigma_{h}$ particles move in either $x>0$ or $x<0$ domain. The horizontal line $S_{h}: 2 \bar{h}_{s}=1$ corresponds to the $\mathbf{U C}$.

It follows from $J=$ const, that

$$
\frac{\partial J}{\partial \bar{\tau}} d \bar{\tau}+\frac{\partial J}{\partial \bar{h}} d \bar{h}=0,
$$

and for the curves that do not cross the $x=0$ plane we obtain

$$
\frac{d \bar{h}}{d \bar{\tau}}=-\frac{\partial J / \partial \bar{\tau}}{\partial J / \partial \bar{h}}=s \frac{1}{2} \frac{\int_{k_{1}}^{k_{2}}\left(2 \bar{h} f^{4 / 3}(k)-1\right) \Psi(k) d k}{\int_{k_{1}}^{k_{2}} f^{2 / 3}(k)\left(-\left(2 k^{2}-1\right)-\bar{\tau} f^{2 / 3}(k)\right) \Psi(k) d k},
$$

where

$$
\Psi(k)=\frac{Q(k)}{f^{4 / 3}(k)} \frac{\left(2 \bar{h} f^{4 / 3}(k)-1\right)^{-1 / 2}}{\left(-s\left(\left(2 k^{2}-1\right)+\bar{\tau} f^{2 / 3}(k)\right)\right)^{3 / 2}}>0 .
$$

It follows from (33), that in the vicinity of an $X$-line the energy of particles which do not cross the $x=0$ plane increases and the particles initially residing in the region $B$ drift to the region $A$. Therefore, the motion of particles in the vicinity of an $X$-line becomes more and more chaotic with time. The phase portrait on the $(\bar{h}, \bar{\tau})$ plane (in other words, solutions of $J=$ const) is shown in Fig. 6a.

On the other hand, in the vicinity of an $O$-line the energy of particles which do not cross $x=0$ decreases and the particles drift from the region $A$ to the region $B$. Therefore the motion of particles in the vicinity of an $O$-line becomes more and more regular. The phase portrait is shown in Fig. $6 \mathrm{~b}$.

The energy of the particles that cross the $x=0$ plane may increase as well as decrease. It can be shown numerically that for any $J$ there is a value of $\bar{\tau}_{J}$ such that $d \bar{h} / d \bar{\tau}<0$ for $\bar{\tau}<\bar{\tau}_{J}$ and $d \bar{h} / d \bar{\tau}>0$ for $\bar{\tau}>\bar{\tau}_{J}$.

\section{Conclusions}

We considered the motion of charged particles in three different regions of the earth magnetotail: in the region with magnetic field reversal and in the vicinities of neutral line of $X$ - and $O$-types. The presence of small parameters (ratio of characteristic length scales in and perpendicular to the equatorial plane and the smallness of the electric field) allows us to introduce the hierarchy of motions. 
We introduced a parameter that plays the role of a measure of mixing in the system. This parameter describes the relative importance of the diffusion of adiabatic invariants on a time scale, defined by the acceleration of particles induced by the electric field. Depending on the value of this parameter, the jumps of the adiabatic invariants destroy original structures in the distribution function or particles accelerate and leave the tail quickly enough to keep the distribution function relatively intact. In the limit of small jumps of the adiabatic invariant we obtained the equations that govern the acceleration of particles. Using this parameter we showed that for particles on long elongated trajectories (small values of $I_{z}$ ) mixing is less prominent than for the rest of the particles.

Our approach illustrates the usefulness of such quantities as quasi-adiabatic invariants (even not perfectly conserved) for the description of particles' motion in the limit when usual guiding center theory breaks down. The developed approach may be useful not only for description of the dynamics of the particles in the magnetotail, but also in other open electromagnetic configurations.

Acknowledgements. This work was performed with the financial support of the Russian Basic Research Foundation Grant No. 0301-00158 and INTAS Grants No. 00-221 (A.N. and D.V.) and 03-513738 (L.Z.) and the Russian Basic Research Foundation Grant No. 04-02-17371 and No. 03-02-16967, and SSh Grant No. 1739.2003.2 (L.Z.). A.N. is also grateful to the Russian Basic Research Foundation Grant No. NSh-136.2003.1 and "Integration" program grant B0053. L.Z. is also grateful to the Humboldt foundation for the support.

Edited by: T. Passot

Reviewed by: two referees

\section{References}

Arnold, V., Kozlov, V., and Neishtadt, A.: Dynamical Systems III. Encyclopedia of Mathematical Sciences, Springer-Verlag, New York, N.Y., 1988.

Ashour-Abdalla, M., Berchem, J., Büchner, J., and Zelenyi, L.: Shaping of the magnetotail from the mantle - global and local structuring, J. Geophys. Res., 98, 5651-5676, 1993.

Benettin, G. and Sempio, P.: Adiabatic invariants and trapping of a point-charge in a strong nonuniform magnetic-field, Nonlinearity, 7, 281-303, 1994.

Bruhwiler, D. and Cary, J.: Diffusion of particles in a slowly modulated wave, Physica D, 46, 265-282, 1989.

Bruhwiler, D. and Zweibel, E.: Energy-spectrum of particles accelerated near a magnetic X-line, J. Geophys. Res., 97, $10825-$ $10830,1992$.

Büchner, J. and Zelenyi, L.: Deterministic chaos in the dynamics of charged particles near a magnetic field reversal, Phys. Lett. A., 118, 395-399, 1986.

Büchner, J. and Zelenyi, L.: Regular and chaotic charged particle motion in a magnetotail-like magnetic field reversals, 1, Basic theory of trapped motion, J. Geophys. Res., 94, 11 821-11 842, 1989.

Büchner, J. and Zelenyi, L.: Regular and chaotic particle motion in sheared magnetic field reversals, Adv. in Space Res., 94, 177$182,1991$.
Burkhart, G., Martin, R., Dusenbery, P., and Speiser, T.: Neutral line chaos and phase space structure, Geophys. Res. Lett., 18, 1591-1594, 1991.

Cary, J. and Skodje, R.: Phase change between separatrix crossings, Physica D, 36, 287-316, 1989.

Cary, J., Escande, D., and Tennyson, J.: Adiabatic invariant change due to separatrix crossing, Phys. Rev., A, 34, 4256-4275, 1986.

Cattell, C., Roth, I., and Linton, M.: The effects of low frequency waves on ion trajectories in the Earth's magnetotail, Geophys. Res. Lett., 22, 3445-3448, 1995.

Chapman, S.: Properties of single-particle dynamics in a parabolic magnetic reversal with general time dependence, J. Geophys. Res., 99, 5977-5985, 1994.

Chapman, S. and Rowlands, G.: Are particles detrapped by constant $B_{y}$ in static magnetic reversals?, J. Geophys. Res., 103, 45974603, 1998.

Chapman, S. and Watkins, N.: Scaling parameters and parametric coordinates in static and time dependent magnetic reversals, Adv. Space Res., 18, 285-289, 1996.

Chen, J.: Nonlinear dynamics of charged particles in the magnetotail, J. Geophys. Res., 97, 15 011-15 050, 1992.

Chen, J. and Palmadesso, P.: Chaos and nonlinear dynamics of single-particle orbits in a magnetotaillike magnetic field, J. Geophys. Res., 91, 1499-1508, 1986.

de Hoffman, F. and Teller, E.: Magnetohydrodymic shock, Phys. Rev., 80, 692-703, 1950.

Deeg, H.-J., Borovsky, J., and Duric, N.: Particle acceleration near X-type magnetic neutral lines, Physics of Fluids B, 3, 26602674, 1991.

Delcourt, D. and Martin, R.: Pitch angle scattering near energy resonances in the geomagnetic tail, J. Geophys. Res., 104, 383-394, 1999.

Elskens, Y. and Escande, D.: Slowly pulsating separatrices sweep homoclinic tangles where islands must be small: an extension of classical adiabatic theory, Nonlinearity, 4, 615-667, 1991.

Itin, A., de la Llave, R., Neishtadt, A., and Vasiliev, A.: Transport in a slowly perturbed convective cell flow, Chaos, 12, 1043-1053, 2002.

Karimabadi, H., Pritchett, P., and Coroniti, F.: Particle orbits in twodimensional equilibrium models for the magnetotail, J. Geophys. Res., 95, 17 153-17 166, 1990.

Kivelson, M. and Russell, C.: Introduction to space physics, Cambridge University Press, Cambridge, 1995.

Landau, L. and Lifshitz, E.: Classical theory of field, Pergamon Press, 1959.

Larson, J. and Tracy, E.: Integrability of charged-particle dynamics in reconnection regions, Phys. Lett. A, 182, 249-254, 1993.

Lyons, L. and Speiser, T.: Evidence for current-sheet acceleration in the geomagnetic tail, J. Geophys. Res., 87, 2276-2286, 1982.

Ma, C. and Summers, D.: Formation of power-law energy spectra in space plasma by stochastic acceleration due to whistler mode waves, Geophys. Res. Lett., 25, 4099-4102, 1998.

Martin, R.: Chaotic particle dynamics near a two-dimensional neutral point, with application to the Earth magnetotail, J. Geophys. Res., 91, 11 985-11992, 1986.

Martin, R., Johnson, D., and Speiser, T.: The energetic ion signature of an O-type neutral line in the geomagnetic tail, Adv. Space Res., 11, 9203-9206, 1991.

Moses, R., Finn, J., and Ling, K.: Plasma heating by collisionless magnetic reconnection: Analysis and computation, J. Geophys. Res., 98, 4013-4040, 1993. 
Neishtadt, A.: Change of an adiabatic invariant at a separatrix, Sov. J. Plasma Phys., 12, 568-573, 1986.

Neishtadt, A.: On the change in the adiabatic invariant on crossing a separatrix in systems with two degrees of freedom, PMM USSR, 51, 586-592, 1987.

Neishtadt, A., Sidorenko, V., and Treshev, D.: Stable periodic motions in the problem on passage through a separatrix, Chaos, 7, 2-11, 1997.

Nocera, L., Pegoraro, F., Bulanov, S., and Bertin, G.: Heating and acceleration at X-point reconnection, Physica Scripta, T63, 197202, 1996.

Northrop, T.: The adiabatic motion of charged particles, Interscience, New York - London - Sydney, 1963.

Petkaki, P. and Mackinnon, A.: Particle orbits near a neutral point, Space Science Reviews, 68, 117-118, 1994.

Savenkov, B., Zelenyi, L., Ashour-Abdalla, M., and Büchner, J.: Regular and Chaotic aspects of Charged particle motion in a magnetotail-like field with a neutral line, Geophys. Res. Lett., 18, 1587-1590, 1991.

Savenkov, B., Zelenyi, L., and Zogin, D.: Motion of particles in thin current sheets, Plasma Phys. Rep., 23, 404-415, 1997.

Smets, R., Delcourt, D., Fontaine, D., and Martin, R.: Structure of electron and ion distribution functions near a reconnection line, J. Geophys. Res., 101, 24 837-24 845, 1993.

Sonnerup, B.: Adiabatic particle orbits in a magnetic null sheet, J. Geophys. Res., 76, 8211-8222, 1971.

Speiser, T.: Particle trajectories in model current sheets, 1, Analytical solutions, J. Geophys. Res., 70, 4219-4226, 1965.

Speiser, T.: Particle trajectories in model current sheets, 2: Applications to auroras using a geomagnetic tail model, J. Geophys. Res., 72, 3919-3932, 1967.
Speiser, T.: Conductivity without collisions or noise, Planetary and Space Science, 18, 613, 1970.

Timofeev, A.: On the constancy of an adiabatic invariant when the nature of the motion changes, Sov. Phys. JETP, 48, 656-659, 1978.

Tsalas, M., Chapman, S., and Rowlands, G.: The stability of charged-particle motion in sheared magnetic reversals, J. Plasma Phys., 65, 331-352, 2001.

Tsyganenko, N.: Global quantitative models of the geomagnetic field in the cislunar magnetosphere for different disturbance levels, Plan. Space Sci., 35, 1347-1358, 1987.

Vainchtein, D., Rovinsky, E., Zelenyi, L., and Neishtadt, A.: Resonances and particle stochastization in nonhomogeneous electromagnetic fields, J. Nonlin. Sci., 14, 173-205, 2004.

Wagner, J., Kan, J., and Akasofu, S.-I.: Particle dynamics in the plasma sheet, J. Geophys. Res., 84, 891-897, 1979.

Wiggins, S.: Adiabatic chaos, Phys. Lett. A, 128, 339-342, 1988.

Wisdom, J.: A perturbative treatment of motion near the $3 / 1$ commensurability, Icarus, 63, 272-289, 1985.

Ynnerman, A., Chapman, S., Tsalas, M., and Rowlands, G.: Identification of symmetry breaking and a bifurcation sequence to chaos in single particle dynamics in magnetic reversals, Physica D, 139, 217-230, 2000.

Zelenyi, L., Zogin, D., and Büchner, J.: Quasiadiabatic dynamics of charged particles in the tail of the magnetosphere, Cosmic Res., 28, 369-381, 1990.

Zhu, Z. and Parks, G.: Particle orbits in model current sheets with a nonzero $b_{y}$-component, J. Geophys. Res., 98, 7603-7608, 1993. 